Pierre-Alain Duc, Jonathan Braine and Elias Brinks, eds.

\title{
Study of Hi and Star Formation Sites in the Magellanic Bridge
}

\author{
Erik Muller ${ }^{1,2}$, Lister Staveley-Smith ${ }^{2}$, William Zealey ${ }^{1}$ \\ ${ }^{1}$ University of Wollongong, Wollongong, Australia \\ ${ }^{2}$ Australia Telescope National Facility, CSIRO, Australia
}

\begin{abstract}
Tidal features represent an important process for the recycling of matter across extremely large spatial scales. The Magellanic Bridge is the closest gaseous tidal feature to our own Galaxy, and offers an ideal opportunity to study the active processes in such phenomena at high spatial resolutions otherwise unavailable. The neutral hydrogen of the western Magellanic Bridge, has been observed with both the Parkes $64 \mathrm{~m}$ telescope and ATCA interferometer and the resulting dataset has revealed an extremely complex and chaotic morphology. We discuss these observations here with reference to a variety of analyses: the spatial correlation of apparently expanding $\mathrm{HI}$ shells with $\mathrm{OB}$ associations and the outcome of sensitive $\mathrm{H} \alpha$ and $\mathrm{CO}$ surveys.
\end{abstract}

The Magellanic Bridge is a filament primarily of neutral hydrogen ( $\mathrm{HI}$ ), extending approximately $22 \mathrm{kpc}$ between the Small and Large Magellanic clouds. The Bridge is clearly kinematically and morphologically contiguous with the SMC and is largely considered to be a tidal feature. This region has recently been observed at $21 \mathrm{~cm}$ wavelength using the ATCA and Parkes Telescopes. A subsequent survey of apparently expanding HI regions in the Bridge has shown that despite an apparently good visual correlation of these objects with the Bridge OB association population (Bica \& Schmitt, 1995), on a more quantitative level, the correlation is very poor. The stellar associations appear to preferentially develop where the integrated $\mathrm{HI}$ column density is $\lesssim 10^{21} \mathrm{~cm}^{-2}$ (Muller et al, 2003).

The young age of the stellar population in the Bridge (Hambly et al. 1994; Grondin et al. 1990), together with the estimated age of the Bridge of $\sim 200 \mathrm{Myr}$ (e.g. Fujimoto \& Sofue 1976), imply that starformation is an active process in the Bridge. To date however, only one molecular region in the Bridge has been detected (Lehner, 2002). Using the SEST telescope, Muller, Staveley-Smith \& Zealey (2003) have located the first CO emission regions in the Magellanic Bridge, detected across three adjacent SEST beams $\left(3 \times \sim 45^{\prime \prime} \sim 40\right.$ pc). The emission region was found to be nearby to a local peak in HI column density and a local peak in $60 \mu \mathrm{m}$ brightness. Empirical models relating the line width and radii of CO regions in the SMC (Rubio, Lequeux \& Boulanger, 1993) suggest that this detected region has a radius of $\lesssim 16 \mathrm{pc}$.

New, sensitive $\mathrm{H} \alpha$ plates have been searched for extended and discrete $\mathrm{Ha}$ emission regions, yielding ten new obvious emission features (Muller \& StaveleySmith, in progress). FAUST objects were found to be within a few tens of 
parsecs of most of the detected $\mathrm{H} \alpha$ features, although a relationship is not always clear. A study of ionisation mechanisms is the subject of future work. Figure 1 shows an uncalibrated map of new $\mathrm{H} \alpha$ objects MBHa4 and MBHa3. These particular features are found nearby to FAUST and IRAS objects and a bright OB association (Bica \& Schmitt, 1995). At this stage, the process responsible for ionisation of the local ISM in either case has not been clearly ascertained.

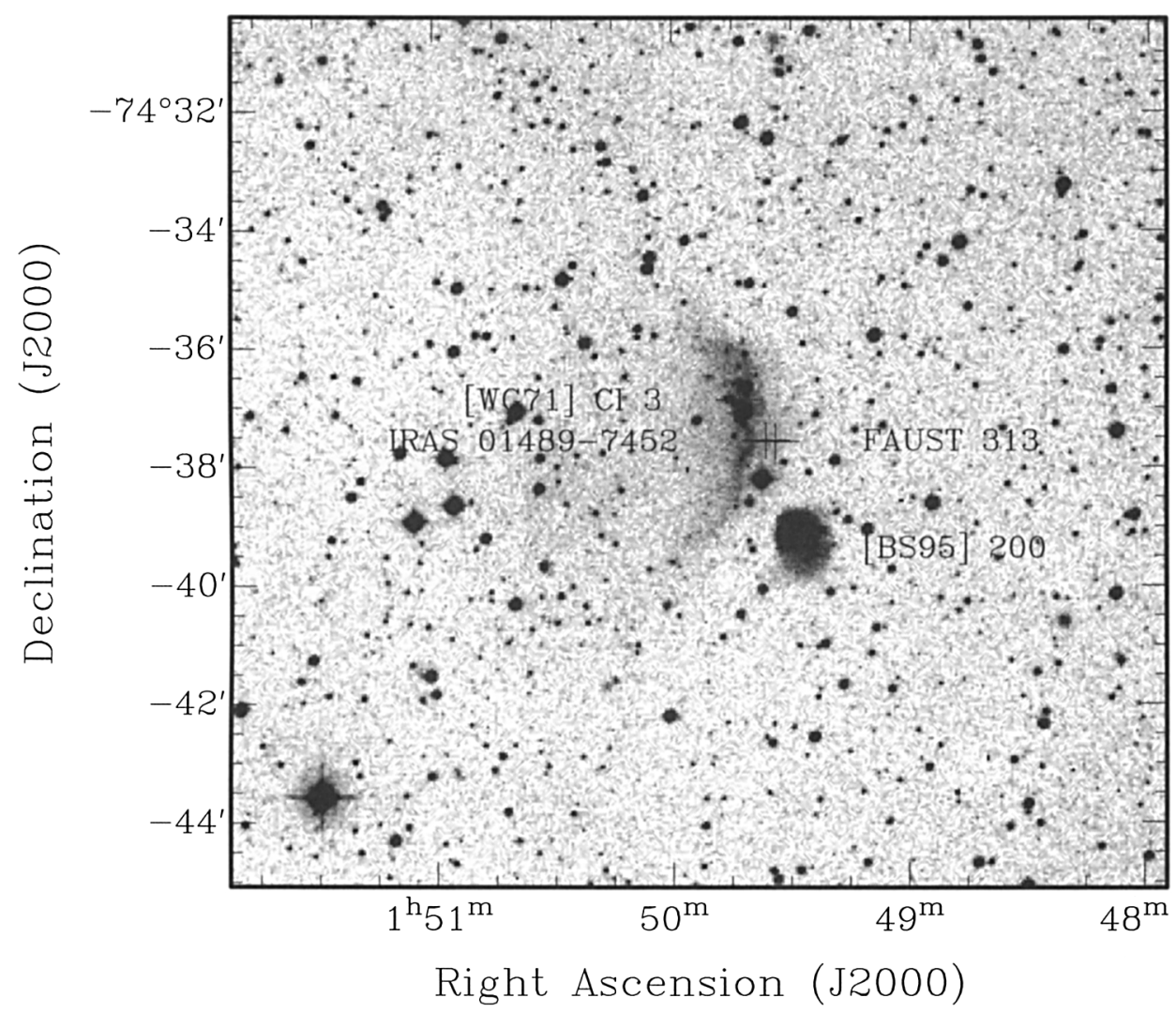

Figure 1. New $\mathrm{H} \alpha$ features MBHA3 (diffuse crescent) and MBHA4 (southern compact region). Nearby objects are marked with crosses.

\section{References}

Bica, E. L. D., \& Schmitt, H. R. 1995, ApJS, 101, 41

Fujimoto, M., \& Sofue, Y. 1976, A\&A, 47, 263

Gardiner, L. T., \& Noguchi, M. 1996, MNRAS, 278, 191 
Gardiner, L. T., Sawa, T., \& Fujimoto, M. 1994, MNRAS, 266, 567

Grondin, L., Demers, S., Kunkel, W. E., \& Irwin, M. J. 1990, AJ, 100, 663

Hambly, N. C., Dufton, P. L., Keenan, F. P., Rolleston, W. R. J., Howarth, I. D., \& Irwin, M. J. 1994, A\&A, 285, 716

Lehner, N. 2002, ApJ, 578, 126

Muller, E., Staveley-Smith, L., Stanimirović, S., \& Zealey, W. 2003, MNRAS, 338,609

Rubio, M., Lequeux, J., \& Boulanger, F. 1993, A\&A, 271, 9 